\title{
Teatro De Fantoches: Uma Apresentação Lúdica De Física Moderna Em Escolas Do Ensino Fundamental.
}

\author{
Puppets Theatre: A Playful Presentation Of Modern Physical Education In Schools Of \\ Fundamental \\ L. F. A. Aringhieri ${ }^{1 *}$; F. A. R. Silva ${ }^{2}$. \\ FAFIS/ICE/Unifesspa, Universidade Federal do Sul e sudeste do Pará, 68500-000, Marabá - Pará, Brasil \\ luizaringhieri@gmail.com
}

(Recebido em 27 de agosto de 2016; aceito em 24 de outubro de 2016)

A proposta de se introduzir os conceitos de Física Moderna no ensino básico é muito instigante, vários estudos já se voltam com esse objetivo, principalmente com o uso de abordagens mais contextualizadas, que envolva os alunos na construção desses conceitos, usando por exemplo, aparatos experimentais através das feiras de ciência, fugindo da metodologia tradicional. O objetivo deste trabalho é apresentar uma metodologia lúdica, através da utilização de bonecos de fantoche e experimentos de baixo custo para disseminar já no ensino fundamental, um pensamento crítico sobre a utilização da tecnologia e as suas consequências para o meio ambiente. Para avaliação da eficácia da proposta foi aplicado um questionário sobre o entendimento do tema antes e reaplicado após da apresentação, visto que um dos objetivos deste trabalho é avaliar o grau de desenvolvimento cognitivo alcançado pelos alunos envolvidos por este projeto, a fim de aprimorar as atividades e técnicas abordadas com os alunos para que estes absorvam com o maior aproveitamento o conteúdo trabalhado. Verificou-se com esse trabalho o envolvimento dos alunos do ensino fundamental a respeito da Física Moderna, associando o conhecimento de forma geral a experimentos elaborados facilitando o processo ensino/aprendizagem. Como resultado obteve-se a melhoria da percepção, a análise critica, e o contexto do mundo ao seu redor.

Palavras-chaves: Bonecos de fantoches 1, metodologia lúdica 2, Física Moderna 3.

The proposal to introduce the concepts of modern physics in basic education is very exciting, several studies show the interest this subject, especially with the use of more contextualized approaches, involving students in the construction of these concepts, using for example, experimental apparatuses through fairs of science, fleeing the traditional methodology. The objective of this paper is to present a playful methodology, using dolls puppet and inexpensive experiments to present yet in elementary school, critical thinking on the use of technology and its consequences for environment. To evaluate the efficacy of proposal was applied one questionnaire on the subject before and reapplied after the presentation, being evaluated the degree of cognitive knowledge gained from the implementation of the project proposal by the students involved in order to improve the activities and techniques explained so that the student absorb and use the better the content worked. Was seen with this work the involvement of elementary school students about modern physics, linking knowledge generally with elaborate experiments facilitating the teaching / learning process. As a result obtained the improvement of perception, critical analysis, and the world context around them

Key-words: Dolls Puppet 1, Playful Methodology 2, Modern Physics 3. 


\section{INTRODUÇÃO}

Ensinar física deveria ser algo mais fácil nos dias de hoje, em virtude do grande número recursos disponíveis aos professores, porém, não é o que se constata, principalmente nas escolas públicas, onde ocorrem várias situações que dificulta a aplicação de um ensino mais significativo influenciando na melhoria da qualidade da relação ensino e aprendizagem [1]. Os recursos didáticos são grandes aliados, pois estimulam o desenvolvimento de habilidades indispensáveis para formação de um cidadão critico, aumentando a percepção dos conceitos [2]. A utilização, pelo professor, apenas de aulas tradicional não basta para que os conteúdos sejam totalmente compreendidos, e a utilização de ferramentas pedagógicas é recomendada pelos Parâmetros Curriculares Nacionais indicando para um aumento da vivência criativas do cotidiano, o desenvolvimento de habilidades motoras, raciocínio lógico, atitudes e capacidades cognitivas que de outra forma não se fariam presentes [3].

Dentre as séries do ensino fundamental, aquela que mais trabalha os conceitos que envolvem os fenômenos da natureza e que contribui para que o aluno possa chegar ao ensino médio com uma bagagem acentuada é o nono ano do ensino fundamental. Nesta série, o aluno, através da disciplina de ciências, passa a estudar e conceituar, fundamentos da física, em consórcio com a química e a Biologia, relação prevista nos PCN's e que se torna algo um pouco difícil ser dissociado por conta superficialidade com que o assunto deve ser abordado [4].

A disposição do conteúdo de física no nono ano, a parte relacionada à física moderna (FM) não é explorada, já no ensino médio, esse tema faz parte do ultimo conteúdo da disciplina no terceiro ano, más infelizmente, o tema, quase sempre, ou não é abordado adequadamente, ou simplesmente não é abordado [5]. Contudo, os alunos vivem inseridos em um mundo com um desenvolvimento tecnológico grandioso, alcançando várias áreas da sociedade, inclusive a educação [6]. A tecnologia está dentro do cotidiano desses jovens, seja nos seus smartphones, nos chips dos cartões, entre outros exemplos. Neste enfoque, é preciso estimular os alunos do ensino fundamental para que eles cheguem ao ensino médio tendo refletido sobre o assunto instigando o professor para apresentar essa realidade de forma mais especifica e daí fundamentando aplicações tecnológicas que influenciam diretamente na vida deles e as suas consequências ao meio ambiente. $\mathrm{O}$ objetivo deste trabalho é apresentar uma metodologia lúdica, através da utilização de bonecos de fantoche e experimentos de baixo custo para disseminar já no ensino fundamental, conceitos muito importantes para física e para sociedade, como é o caso da física moderna. Valendo-se do seu grande poder de assimilação e desenvolvedor cognitivo, o teatro de fantoches irá desmitificar e por fim aos problemas encontradas na maioria das salas de aulas ao longo do ensino médio, atraindo a atenção dos alunos com uma linguagem apropriada usando temas inerentes para a sua inserção nesse mundo tão interessante e dentro de sua faixa etária.

\section{MATERIAL E MÉTODOS}

O trabalho consiste na montagem de um cenário caracterizado em cima de três conteúdos de física moderna que devido a sua complexidade não são trabalhados no ensino fundamental, más que fazem parte da vida cotidiana dos alunos desse nível escolar. Assuntos como o de Ótica, em que contextualiza a forma de como o olho humano enxerga, a natureza da luz, a decomposição das cores, e a luz como forma de energia. A Teoria da Relatividade, em que 
apresenta aos alunos o significado do movimento quando comparado com algum outro ponto de referência, a característica da velocidade da luz, o paradoxo dos gêmeos, e a geometria espaçotempo que são fundamentais para apresentação da física quântica, e a Cosmologia, que introduz os conceitos sobre a origem, estrutura e evolução do Universo a partir da aplicação de métodos científicos.

Para o desenvolvimento dos conteúdos, foram elaborados roteiros teatrais através de "historinhas" com seu teor adaptados em uma linguagem que proporcione a melhor assimilação dos conteúdos associado com experimentos que relacionam o tema a apresentação, e que contemple a interação constante e direta com o público-alvo. A participação dos alunos no contexto da apresentação é de fundamental importância para condução da dinâmica do trabalho, já que, a partir das indagações e questionamentos propostos é que serão apresentados os fundamentos teóricos e a aplicação dos experimentos com o objetivo de consolidar a aprendizagem.

Para execução das apresentações teatrais foram confeccionados 4 bonecos, que usados por seus interlocutores, desenvolveram os roteiros elaborados a partir de fatos do cotidiano despertando o interesse de como esses fatos acontecem, e quais os seus fundamentos.

Primeiro (Elaboração do texto e roteiro): para desenvolver esta proposta o professor orientador deve limitar/escolher o tema a ser trabalhado em sala. Cabe ressaltar que esta metodologia serve para todas as disciplinas, não só para a Física, bastando ser adaptada à realidade e ao teor de cada disciplina. Após a escolha do tema, deverá ser elaborado o roteiro das historias com as falas dos personagens. Neste momento, a equipe deve observar e escolher os experimentos apropriados para o tema usado no trabalho e inserir a realização desses experimentos de forma sincronizada ao desenvolvimento do conteúdo do texto trabalhado, de forma que sejam trabalhados até o fim da apresentação.

Segundo (Aquisição de materiais): deve-se fazer o levantamento geral dos materiais necessário para a apresentação. Esta fase se ocupará desde o sistema de sonorização até os materiais utilizados para a realização dos experimentos. Na maioria das vezes, escola possui toda a logística de som e mídias (caixa de som, micro fones, note book e data show) o que nos permite afirmar e garantir a viabilidade de aplicação desta metodologia de ensino.

Neste passo deverão ser verificados os materiais que serão usados para os experimentos, para o cenário (estrutura, lona ou banner com layout do cenário), os bonecos que podem ser adquiridos pela internet ou confeccionados artesanalmente e a montagem da apresentação, assim como a parte de simuladores, imagem e vídeos, em slides. Ou seja, nesta etapa é confeccionada a apresentação. É importante não se esquecer de fazer o layout do banner/lona (fachada do cenário) usando figuras e imagens inerentes ao assunto que está sendo trabalhado de forma que sugira a reflexão ao tema.

Terceiro (cenário): a estrutura do cenário onde a lona ou o banner $(2.5 \mathrm{~m} \times 2,0 \mathrm{~m})$ é fixado foi confeccionada em estrutura metálica com tubo de $3 / 4$ ", porém pode ser feita com tubo de $3 / 4$ " de pvc, em madeira ou conforme a disponibilidade de recursos disponíveis na comunidade escolar, conforme sua criatividade.

Quarto (avaliação): esta etapa se restringe ao professor, pois deve ser confeccionado um questionário sobre o tema proposto, o qual tem a finalidade de mensurar o conhecimento da turma com relação ao assunto escolhido. Trata-se de uma prova objetiva contendo 10 questões 
múltipla escola. Esta avaliação deve ser aplicada em dois momentos, um dia antes da apresentação e o mesmo questionário deve ser aplicado após a apresentação. Assim o professor consegue realizar em estatística referente ao ganho percentual na assimilação do conteúdo, pautando-se no desenvolvimento adquirido pela média aritmética da turma.

A figura 1, a seguir, apresenta os bonecos "Solzão, Solzão (com mais idade), Dalvinha, e Solzinho", na sequência da esquerda para direita. O fato de haver dois bonecos, o Solzão e o Solzão (com mais idade), ambos com o mesmo figurino, é proposital, para que o interlocutor discorra sobre o paradoxo dos gêmeos, ou seja, o mesmo personagem após a viagem do seu irmão gêmeo ao espaço. Além dos bonecos e do público alvo a apresentação conta com participação do professor, tratando-se da pessoa envolvida no projeto e que tem o conhecimento dos fundamentos para explicação mais específica dos fenômenos usando como ferramenta os experimentos de baixo custo, simuladores computacionais e contextualizações apresentadas em power point .

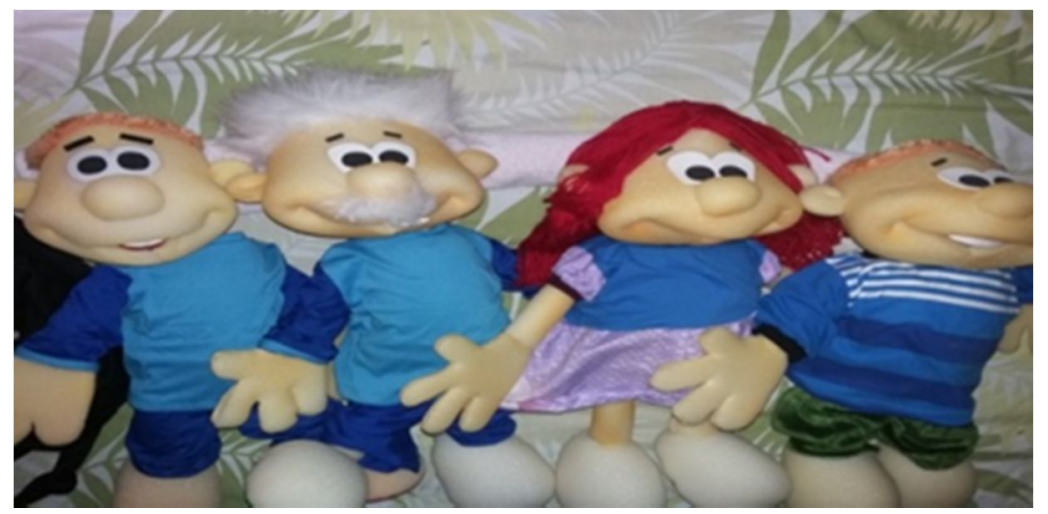

Figura 1. - Bonecos de fantoche usados na apresentação do teatro.

Outra ferramenta importante na execução da proposta é o uso de experimentos que são voltados para o entendimento de cada tema proposto. Sobre o tema que trata dos fundamentos de ótica serão usados os experimentos do disco de Newton (figura 2), Luminescência (figura 3) e efeito fotoelétrico (figura 4).

No Disco de Newton serão utilizados materiais como: folha de papel branca, compact disc (CD), cola, tesoura, lápis colorido, régua e ventilador. O objetivo é demonstrar a decomposição da Luz Branca formada a partir de cores primárias. Na figura 2 temos na sequência da esquerda para direita, o material usado para elaboração do experimento, a composição do disco com as cores primárias e execução do experimento com ajuda do ventilador. 

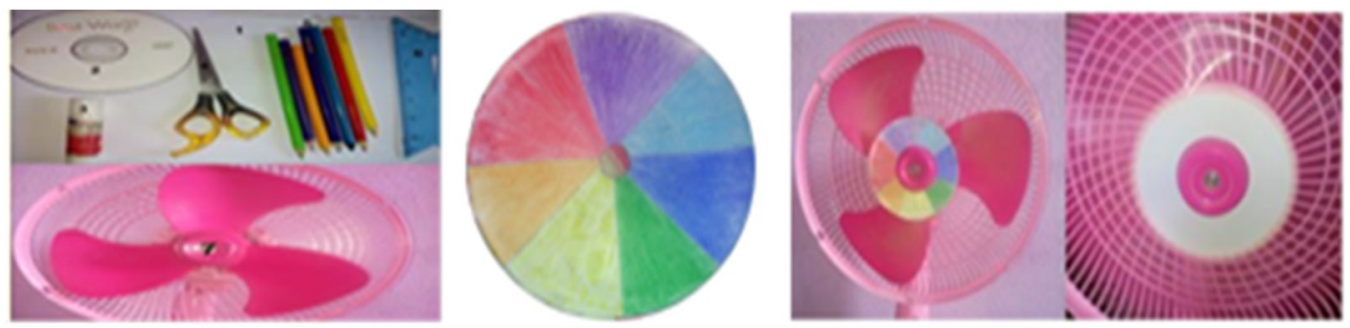

Figura 2. - Experimento do Disco de Newton.

Outro experimento é o da Luminescência, nesse experimento o objetivo é apresentar aos alunos a percepção da radiação, ou seja, como alguns materiais emitem energia após serem excitados, fenômenos presente, por exemplo, nas comunicações, microondas, ou na medicina. Para esse experimento, foi utilizado tinta acrílica fluorescente que simulava alguns materiais que passam a emitir brilho intenso após serem excitada pela luz negra. Para execução da experiência tem-se como material: lâmpada de luz negra, recipientes transparentes, água, canetas hidrocor, sabão em pó, objetos fluorescentes, soquete de lâmpada, e tesoura. A figura 3 apresenta os materiais usados e os resultados esperados na execução da experiência.
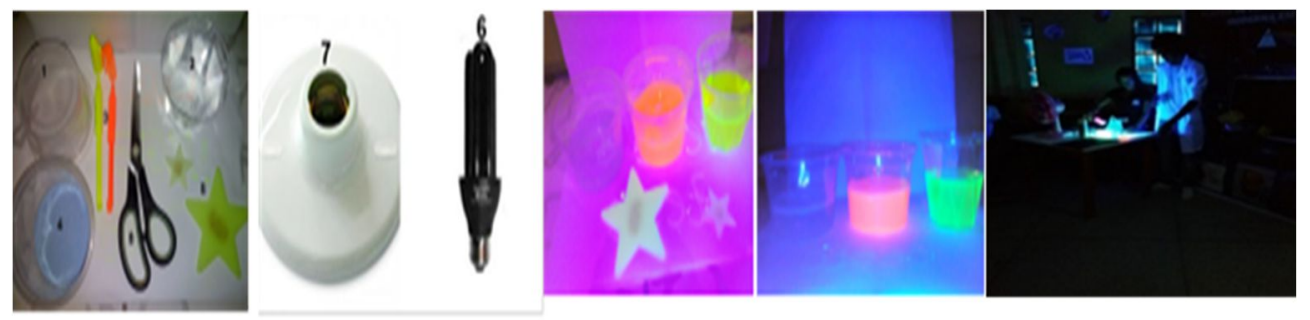

Figura 3. - Execução do experimento da luminescência.

Para o entendimento sobre o conceito da energia promovido pela luz foi realizado o experimento sobre o Efeito Fotoelétrico. O intuito foi demonstrar a emissão de elétrons de um material, geralmente metálico, quando ele é submetido à radiação eletromagnética (no caso, incidência de luz), e exemplificar a larga aplicação no cotidiano como, por exemplo, a contagem do número de pessoas que passam por um determinado local, o sistema de acendimento da iluminação pública entre outros. $\mathrm{Na}$ construção do experimento foram usados materiais como, resistor dependente de luz (LDR), fios, bateria $3 \mathrm{~V}$, chave liga/desliga e um suporte de madeira, respectivamente da esquerda para direita, conforme ilustra na figura 4. Para complementar a apresentação desse conceito, o efeito fotoelétrico foi trabalhado com o uso de simuladores computacionais didáticos e slides em Power Point. 

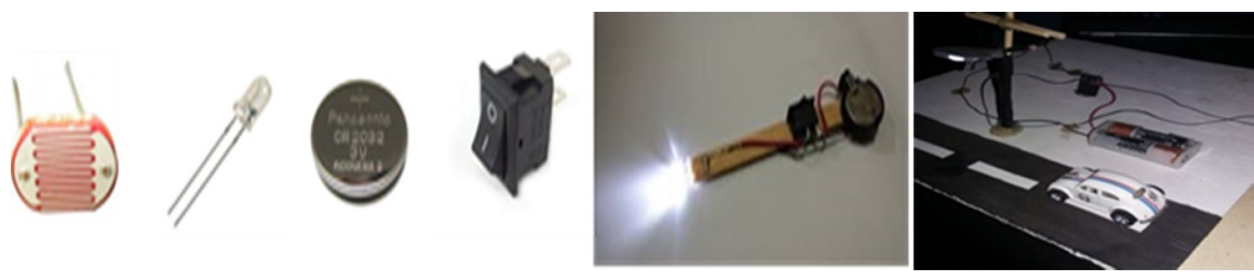

Figura 4. - Objetos e execução do experimento do efeito fotoelétrico.

$\mathrm{Na}$ apresentação de Cosmologia, os conceitos de origem, estrutura e evolução do Universo foram trabalhados no contexto da apresentação dos bonecos. Um experimento realizado junto com a apresentação contempla a teoria do Big Bang, em que o principal objetivo é demonstrar que o universo não é estático e se encontra em constante expansão, ou seja, as galáxias estão se afastando umas das outras [7]. Para a demonstração dos conceitos sobre a expansão do Universo foram usados balões de festa, pedaços de papel colorido, e cartolina preta. A figura 5 apresenta o material e a demonstração do experimento que representa a Teoria do Big Bang dentro do contexto.

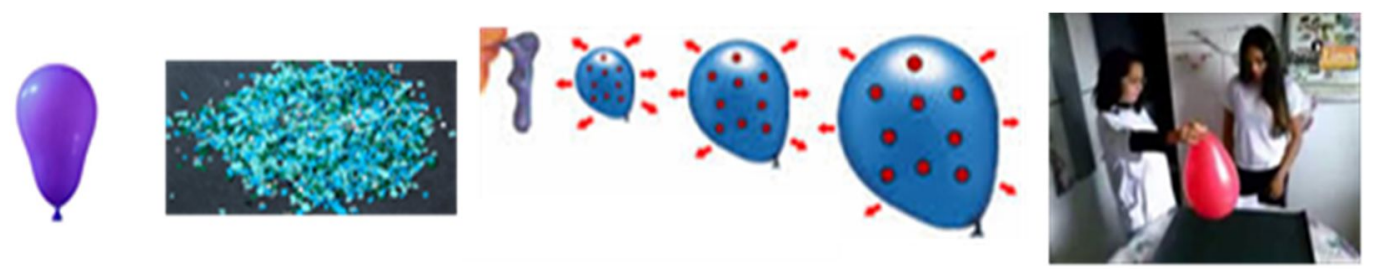

Figura 5. - Apresentação do material, demonstração de execução do experimento.

A parte do trabalho que trata da relatividade foi abordada conforme duas metodologias, uma delas utilizando os bonecos de fantoches, abordando a parte que trata da Teoria dos Gêmeos. Nesse caso, para explicar o paradoxo dos gêmeos foi feito uma representação da viagem de um dos bonecos ao espaço na velocidade da luz, voltando para casa mais novo do que o gêmeo que ficou em Terra, movendo-se a velocidades menores. Nesse caso, o boneco que ficou na Terra se apresenta com uma aparência de mais idade, a fim de mostrar a ocorrência da teoria junto aos alunos. Para complementação da explicação da teoria foi apresentado através de Power Point uma breve fundamentação teórica através da exposição de vídeo referente ao assunto.

A figura 6 apresenta a outra metodologia usada para demonstração da Teoria da Relatividade, em que, foi elaborada através da simulação da deformação (ou curvatura) do espaço-tempo provocada por uma massa, demonstrando a natureza geométrica da interação gravitacional, onde serão utilizados os seguintes materiais: tecido de algodão e uma bola. 


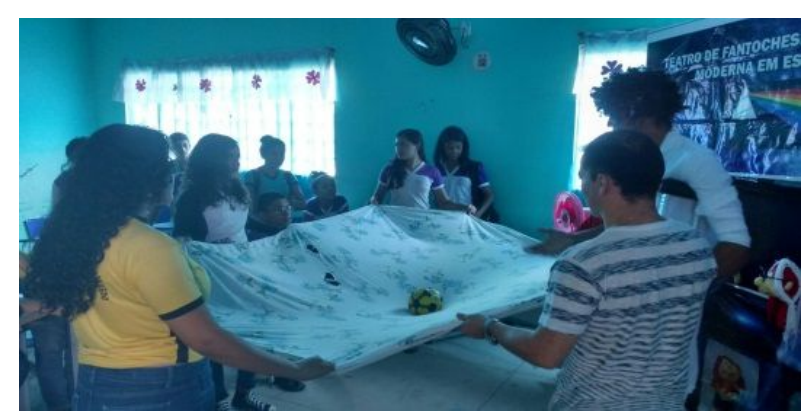

Figura 6. - Execução da atividade experimental com ajuda dos alunos sobre a deformação espaço/tempo.

Para mensurar o desenvolvimento cognitivo dos alunos, e a eficiência da aplicação do projeto, foi realizado a aplicação de um questionário antes e após as apresentações. Na composição do questionário foram elaboradas questões de cunho interpretativo, tendo como foco cada conceito a ser abordado estando relacionado às situações do cotidiano dos alunos.

Para aplicação do projeto foram escolhidas quatro escolas de ensino fundamental da rede pública de ensino do município de Marabá-PA. Foram realizadas reuniões prévias com a equipe pedagógicas de cada escola e foi determinado que seria usado a carga horária de duas aulas da disciplina de ciências com a intenção de avaliar a aplicabilidade da proposta metodológica em uma carga horária normal do professor da disciplina.

\section{RESULTADOS E DISCUSSÃO}

Para o desenvolvimento do trabalho não houve qualquer tipo de resistência das equipes pedagógicas das escolas. Todas as apresentações contaram com a disponibilização dos ambientes e equipamentos necessários para sua aplicação. Além das equipes pedagógicas, os alunos foram muito receptivos a forma como foi apresentado temas que são apresentados em outros níveis de ensino de forma bem mais complexa. O resultado da interação dos alunos com os bonecos estava estampado no comportamento entusiástico que foram expostos. A interação com os bonecos deram margem a uma dinâmica muito apropriada já que os alunos não ficaram reprimidos, todos riam nos momentos mais descontraídos e todos silenciavam nos momentos que era necessário ter uma maior concentração. Pode se colher também expressões escritas na forma de recados escritos nos questionário que foram usados para medir o entendimento sobre o assunto. Recados com expressões de agradecimento sobre a inovadora metodologia de como foi discutido a ciência do cotidiano.

Para mensurar quantitativamente, foram coletados dados através das aplicações dos questionários aos alunos, dentro de um rol de quatro escolas de ensino fundamental da rede pública de ensino do município de Marabá, onde foi obtido o desempenho dos alunos de cada escola, e que estão representados na figura 7. O desempenho foi expresso em termos da nota média da turma tendo como contexto as 10 questões apresentadas no questionário. Foi avaliado o desempenho dos alunos através dos questionários respondido antes da apresentação (vermelho) e o desempenho após a apresentação (azul). No gráfico, em termos percentuais também é apresentado o rendimento final da aplicação do projeto. 


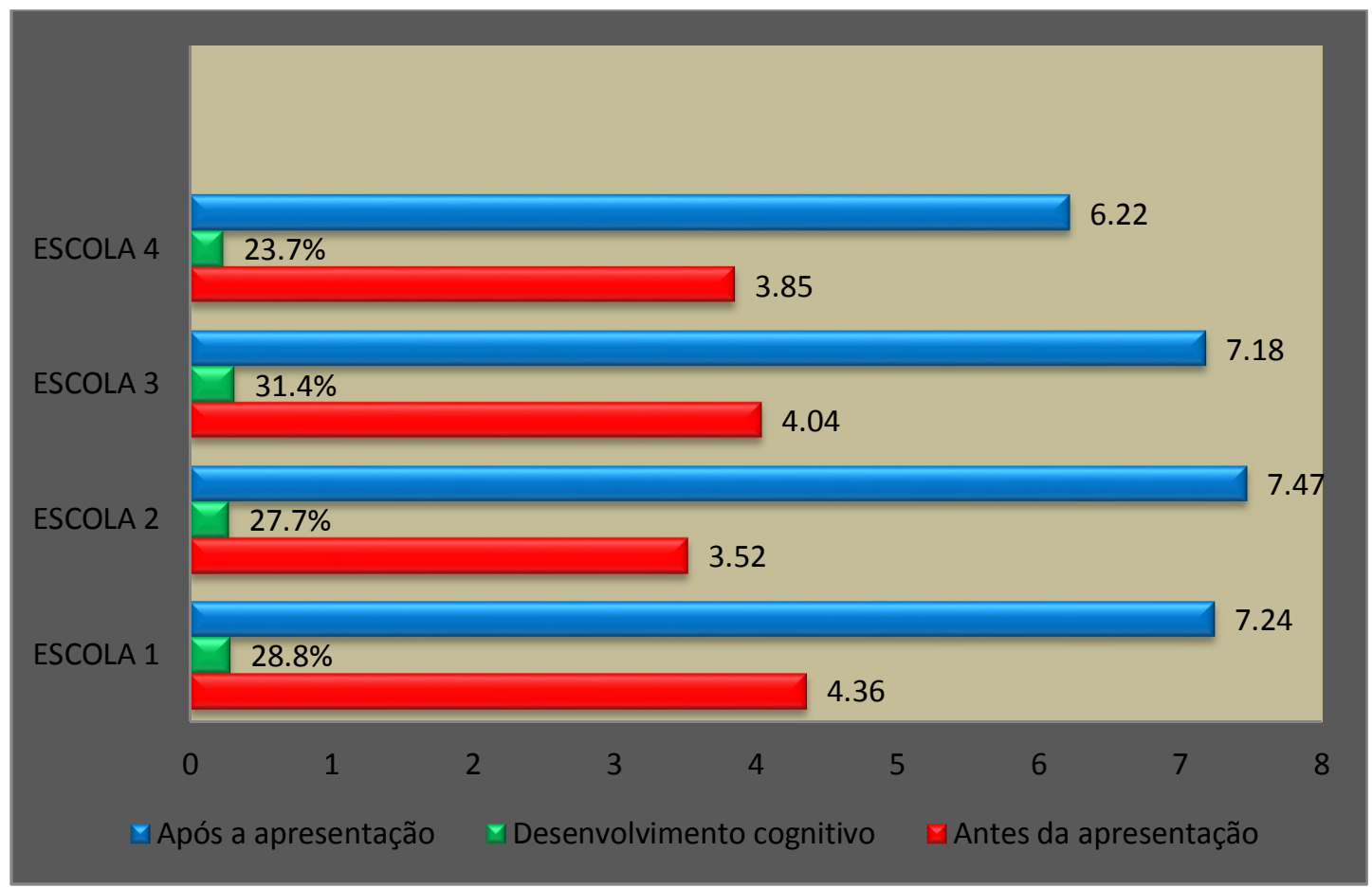

Figura 7. - Gráfico que apresenta de forma quantitativa a aplicação do trabalho.

Com a avaliação do desenvolvimento cognitivo obtido pode-se verificar um rendimento médio de quase $30 \%$ de aproveitamento, resultado que valida a aplicação da metodologia para o nível de ensino objeto da pesquisa. Através dos objetivos apresentados foi muito proveitoso a participação dos alunos da graduação de física licenciatura que tiveram a oportunidade de envolvimento com a abordagem de assuntos de física moderna em sala de aula em uma situação real de docência. Através desta participação foi efetivado um vinculo muito importante entre a universidade e as escolas de ensino básico viabilizando o processo de otimização dos recursos didáticos em desenvolvimento no ambiente acadêmico das instituições de ensino superior.

A figura 8 apresenta algumas fotos obtidas nos momentos de apresentação nas escolas. Um fato curioso é que não foi atingido o objetivo da participação dos professores de ciências, titulares das escolas na aplicação do projeto. $\mathrm{Na}$ aplicação do projeto estes profissionais não compareceram as apresentações. Desta forma não teve como avaliar a aplicabilidade da proposta do trabalho pelo ente que é responsável e conhece os alunos dentro da sua dinâmica, na sua rotina diária, o que seria importante para serem avaliadas as diversas reações apresentadas pela aplicação de uma nova metodologia.

Através da aplicação do projeto pode se afirmar a mudança de comportamento dos alunos quando os conteúdos são apresentados de uma forma contextualizados, o teatro com bonecos, o uso de experimentos de baixo custo ou experimentos mais elaborados fazem parte de metodologias que atraem a participação, a inserção de questionamentos individuais e personalizados. Assim, a física se torna uma ciência muito mais atrativa instigando o auto desenvolvimento, a busca pelo conhecimento passa ser um objetivo de entendimento do seu diaa-dia. 

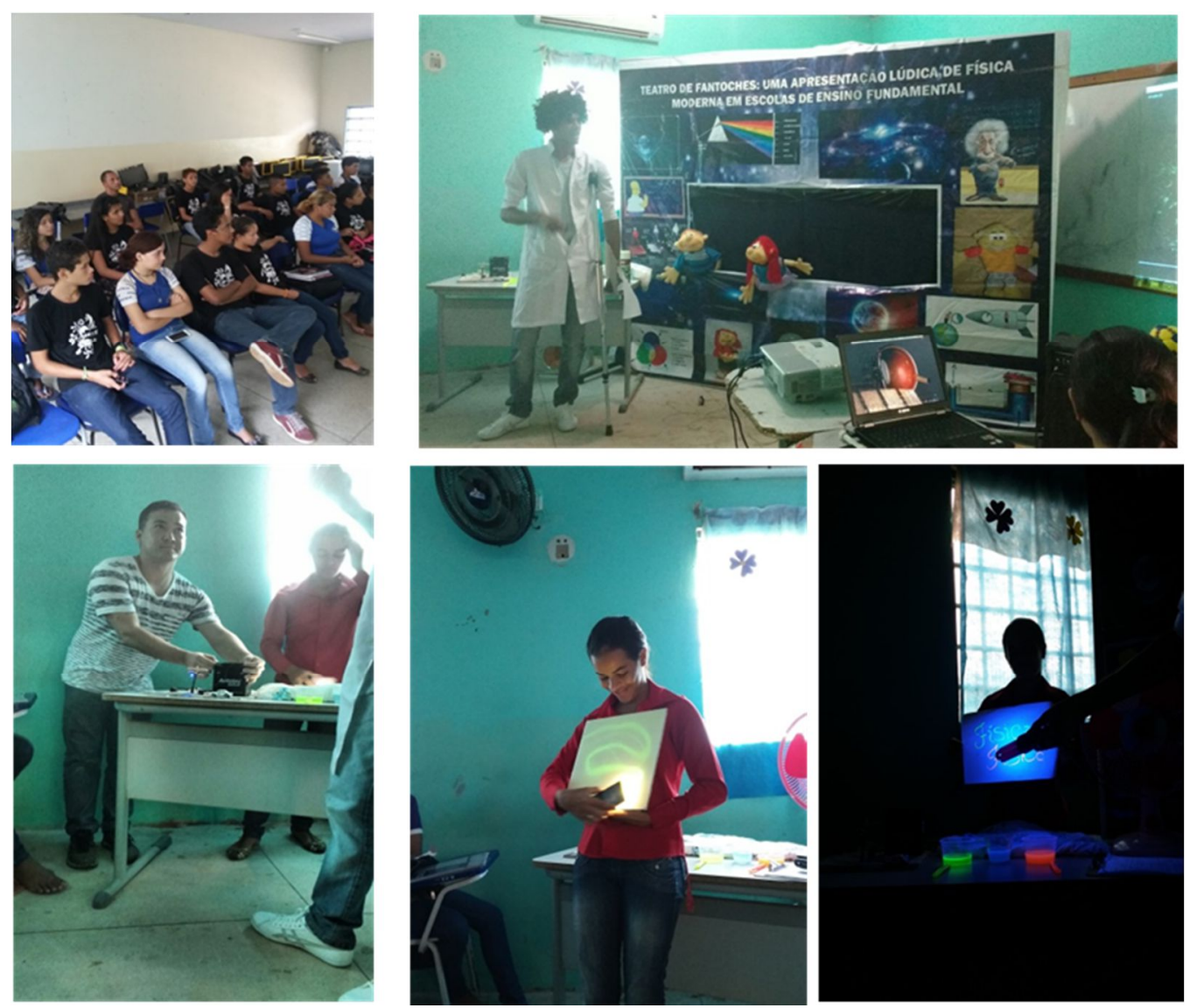

Figura 8. - Alguns momentos das aplicações do projeto nas turmas de nono ano do ensino fundamental da rede pública no município de Marabá-PA.

\section{CONCLUSÃO}

A integração entre prática experimental, mídias, teatro de fantoche e pedagogia, é perfeitamente viável na aplicação do conhecimento de forma geral. Em física, o conhecimento se torna mais palpável, mais acessível, assuntos como o de física moderna, tratados de forma complexa associados com requisitos que não estão muitas vezes relacionados com o cotidiano dos alunos, más que, a partir da aplicação do projeto pode se ampliar a percepção dos alunos do ensino fundamental sobre vários temas que envolvem as novas tecnologias bastante difundidas na sociedade moderna. Foi proporcionada uma forma prazerosa de desenvolver as habilidades de observação, de ouvir, de expressar-se com clareza e objetividade de transmitir e receber informações dentro do contexto de Física Moderna. Temas como as características da luz, que a partir da sua relação com o conceito de uma onda eletromagnética e o conceito de fótons de luz mudou o entendimento sobre a natureza, ou mesmo as novas concepções do movimento trazida pela Teoria da Relatividade, e finalmente uma leitura sobre a evolução do universo foram tratados de forma bem simples melhorando o desempenho na visão qualitativa e quantitativa que envolve a tríade Ciência, Tecnologia e Sociedade (CTS). A participação dos alunos das escolas de ensino fundamental do município de Marabá no projeto foi muito importante, essa interação foi estimulante e comprovou de maneira satisfatória a aplicabilidade de uma pedagogia significativa. Pode comprovar também a viabilidade da relação institucional entre a escola pública e a universidade estreitando o vinculo do desenvolvimento de novas metodologias de ensino viabilizando uma maior amplitude do conhecimento. 


\section{AGRADECIMENTOS}

Ao Mestrado Nacional Profissional em Ensino de Física promovido pela Sociedade Brasileira de Física (SBF), polo Universidade Federal do Sul e Sudeste do Pará.

\section{REFERÊNCIAS BIBLIOGRÁFICAS}

1. Silva D, Pinheiro EE, Macedo G ME, Lima BRA, Silva RAC, Sousa TO. A utilização de recursos didáticos por professores de escolas públicas de Teresina-PI.. 63 ${ }^{a}$ Reunião Anual da SBPC. G. Ciências Humanas-7 edição- 11. Ensino-Aprendizagem. Setembro 2010.

2. Scorsatto MC, Dullius MM, Konrad O. Uma Abordagem Alternativa para o Ensino da Física: Consumo Racional de Energia. Textos de Apoio ao Professor de Física, v.17 n.2, 2006. Instituto de Física - UFRGS. Porto Alegre, julho 2006.

3. Maria CAL. Medições Docentes em Fóruns Virtuais. $1^{\mathrm{a}}$ Edição. Belém, PA. Editora EDITAEDI, 2015. $35-50 \mathrm{p}$.

4. Zabala A. Enfoque Globalizador e Pensamento Complexo: uma proposta para o currículo escolar. Porto Alegre: ARTMED Editora, 2002.

5. Araujo MST, Abib MLVS. Atividades Experimentais no Ensino de Física: Diferentes Enfoques, Diferentes Finalidades. Revista Brasileira de Ensino de Física, vol. 25, no. 2, Junho, 2003.

6. Medina M, Braga M. O Teatro como Ferramenta de Aprendizagem da Física e de problematização da Natureza da Ciência. Cad. Bras. Ens. Fís., v. 27, n. 2: p. 313-333, ago. 2010. http://dx.doi.org/10.5007/2175-7941.2010v27n2p313.

7. Machado KD. Teoria do eletromagnetismo. Volume II. Ponta Grossa, PR. Editora UEPG, 2002. $825 \mathrm{p}$. 